\title{
MICROVASCULAR SURGICAL TECHNIQUES USED TO PROVIDE SKIN COVER OVER AN UNUNITED TIBIAL FRACTURE
}

\author{
Robert Acland, Louisville, United States of America and Paul Smith, Glasgow, Scotland
}

\begin{abstract}
A case is described in which a large area of unstable skin overlying a tibial fracture was replaced by a single stage skin flap transfer, using microvascular surgical techniques. The shortening of treatment time and the improvement in the local blood supply compared to that provided by orthodox skin flaps are noted. The improved blood supply at the fracture site may have encouraged bony union.
\end{abstract}

The problem of the compound tibial fracture with overlying skin loss is familiar to all orthopaedic surgeons. When the soft-tissue damage is extensive, the need for good skin cover over the fracture site sooner or later arises. Local skin is often inadequate both in quality and in quantity, and therefore a distant flap is usually needed. By standard methods, this may either be taken from the opposite leg or it may be migrated from the abdomen in multiple stages as a tube. These standard techniques have numerous disadvantages because they are technically difficult, time consuming, uncomfortable for the patient and sometimes unsuccessful. Further, the patient is away from the care of the orthopaedic surgeon for a long time.

The concept and use of axial pattern skin flaps and recent advances in microvascular surgery have produced a new technique of immediate skin flap transfer.

An axial pattern flap (McGregor and Jackson 1972) is one which is based on a known arteriovenous axis. It is thus a functionally self-contained vascular territory, and depends for its blood supply on vessels with an external diameter of one or two millimetres. Now that it is possible to anastomose vessels as small as this with dependable success (Acland $1972 a$ and $b$; Hayhurst and O'Brien 1975), an axial skin flap may be freely transplanted to any area in which suitable recipient vessels can be found (Daniel and Taylor 1973; Harii, Ohmori and Ohmori 1974). This paper presents a case which illustrates the advantages of this technique of "free flap" transfer.

\section{CASE REPORT}

A thirty-six-year-old man presented with a chronically infected ununited tibial fracture associated with a painful disorganised ankle joint and a large unstable scar adherent to the tibia. In July 1974 he had fallen twenty feet and sustained a severely comminuted fracture of the lowermost third of the tibia, with upward displacement of the talus through the tibial articular surface. Manipulation was unsatisfactory and internal fixation with screws was done through an antero-medial incision. This also failed and the disorganisation of the ankle joint persisted. Two months later there was a severe wound infection.

After further conservative management the patient was referred to the Western Infirmary, Glasgow. It was then nine months after the injury, the fracture was ununited and there was clinical evidence of infection, although the wound was superficially healed. Radiographs confirmed the presence of non-union, with sclerosis of the bone ends, cavity formation and sequestration.

At operation in April 1975 sequestrectomy was done and the screws were removed. Healing was slow and a chronic discharging sinus developed, communicating with a cavity at the tibial fracture site. The sinus closed in response to antibiotic treatment, but the cavity persisted. It was thought that

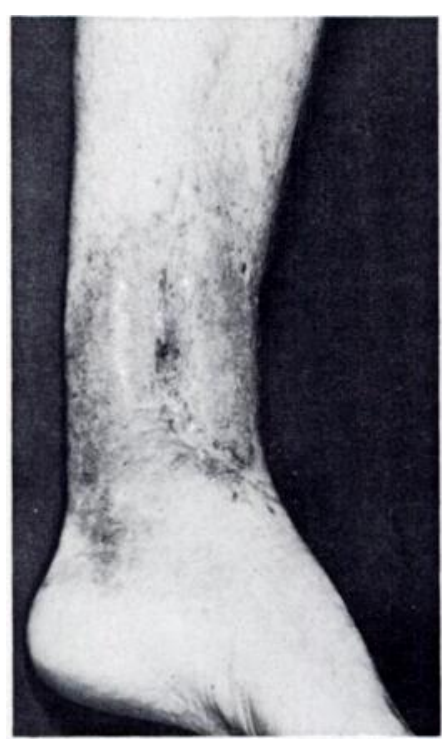

Fig. 1

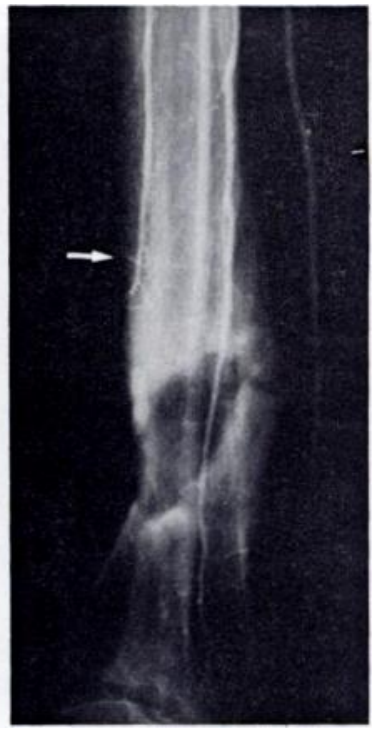

Fig. 2
Figure 1-The appearance before operation showing scarred unstable skin over the fracture site. Figure 2-The angiograph before operation. The arrow shows the end of the anterior tibial artery which was used to supply the flap.

the patient needed bone grafting of the tibial fracture and fusion of the ankle joint, but the condition of the overlying skin was too poor to permit these procedures (Fig. 1). A posterior approach to the anteriorly placed cavity was not indicated, because it would have entailed damaging the only contact area between the tibial fragments.

Replacement of the overlying skin with a skin flap was therefore indicated, and a free flap transfer was planned.

Femoral arteriography (Fig. 2) showed that there were suitable vessels near the recipient area. The posterior tibial

R. D. Acland, F.R.C.S., The Microsurgery Laboratory, Division of Plastic and Reconstructive Surgery, University of Louisville, Kentucky 40201, United States of America.

P. J. Smith, F.R.C.S., Orthopaedic Department, Western Infirmary, Glasgow, Scotland. 
artery was intact, as was the peroneal. The anterior tibial artery ended abruptly near the perimeter of the scarred area. Suitable superficial recipient veins were visible on direct inspection. The groin was chosen as the donor site for the flap. Operation-The operation was in July 1975. The intended margin of the scar excision (measuring 18 by 11 centimetres) was marked out on the leg, and a paper template of the marking was made. From the upper end of the scarred area an exploratory incision was made upwards and laterally. Through this, a superficial vein and the anterior tibial artery were exposed.

Using the template, the outline of the flap was drawn in the groin. Its long axis was centred on the known course of the superficial circumflex iliac artery and vein. Its medial end was just lateral to the femoral artery.

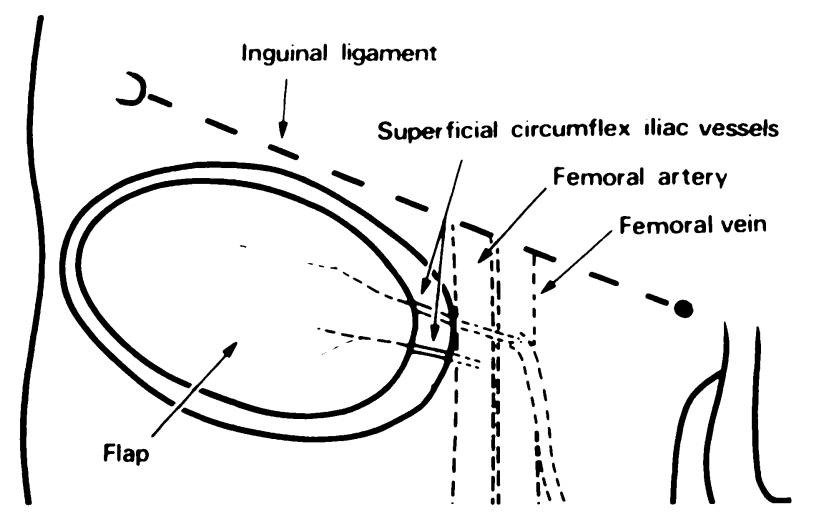

FIG. 3

The anatomy of the free groin flap, supplied and drained by the superficial circumflex iliac vessels.

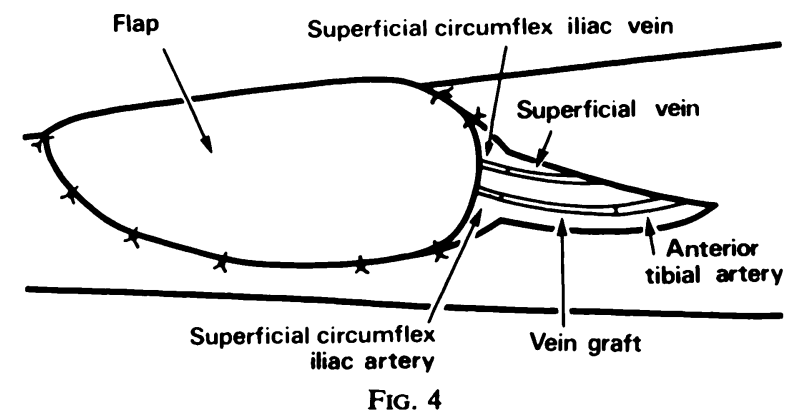

The flap transferred to the leg, with anastomosis of the flap vessels to the anterior tibial artery and a neighbouring superficial vein.

The medial edge of the groin flap margin was incised. The superficial circumflex iliac artery and vein were identified in the subcutaneous fat and traced to their origin from the femoral vessels. The remainder of the flap margin was incised. The flap was raised to include the full thickness of the subcutaneous fat and also the deep fascia of its medial half (Fig. 3). The flap was then left for thirty minutes, attached to the body only by the two vessels and two skin sutures. At the end of this period it showed a normal colour and capillary return.

The area of scarred skin was excised from the leg and haemostasis was obtained. The fracture site was obviously ununited and the presence of a large cavity in the tibia was confirmed.

The superficial circumflex iliac artery and vein were now ligated at their origin and divided. The flap was moved to the leg and loosely sutured in position. The vein of the flap was anastomosed end-to-end to the superficial recipient vein by using eight interrupted sutures of $11 / 0$ monofilament nylon (Acland $1972 a$ and $b$ ). Both veins were two millimetres in external diameter. The anterior tibial artery was cut back until good blood flow was obtained. This produced a gap of three centimetres between the anterior tibial artery and the artery of the flap. A suitable vein graft of matching diameter was taken from the foot, reversed and anastomosed at each end to join the two arteries (Figs. 4 and 5). The diameter of the anterior tibial artery was 2.5 millimetres and that of the artery of the flap was 1.5 millimetres. On release of the clamps there was brisk bleeding from the margin of the flap and a rapid return of colour. Suture of the flap margin was completed and the groin wound was closed by direct suture. Progress-After operation the leg was elevated on pillows. No dressing was applied. No anticoagulant or antithrombotic treatment was given. A broad spectrum antibiotic was given for seven days in view of the underlying bony cavity. Healing was uneventful (Fig. 6). The patient was allowed out of bed at ten days; he was fully ambulant at fourteen days and was returned to the care of the orthopaedic department.

Five weeks after the free fiap transfer he was readmitted for bone grafting. However, clinical examination now revealed a solidly united fracture and tomography demonstrated that the bony cavity was beginning to fill with new bone. He was therefore allowed to discard the caliper and to bear weight freely. Arteriography at eight weeks (Fig. 7) confirmed the patency of the vascular anastomosis.

\section{DISCUSSION}

Our patient, a year after injury, had an ununited fracture, a disorganised ankle joint and poor skin cover requiring flap replacement. Conventional transfer of a multi-stage skin flap as a necessary preliminary to any orthopaedic procedure would have taken on average five months (Stranc, Labandter and Roy 1975). Because of the prospect of such a long and uncertain course of treatment, the question of amputation had been raised but refused by the patient.

The use of an immediate skin flap transfer in this patient's case had clear advantages over conventional methods. It dramatically shortened the treatment and eliminated the need for periods of uncomfortable fixation and difficult nursing. The groin has two advantages as a free flap transfer. The anatomy of the cutaneous vessels arising in the groin is well established and dependable (Smith, Foley, McGregor and Jackson 1972), and the defect produced by taking the flap can be closed by direct suture, leaving an unobtrusive scar.

We were struck by the rapid development of bone union after the flap transfer. It is arguable that union would in any case have occurred at this time, although this seems unlikely in view of the previous slow progress: Even if this particular fracture was one which would have ultimately progressed to union, albeit slowly, the impression remains that the increased blood supply to the area around the fracture site had a beneficial influence on the rate of union.

Plastic surgeons are familiar with the fact that bony union may follow the application of a conventional skin flap to a fracture site (Connelly 1948, 1956; Grabb and 


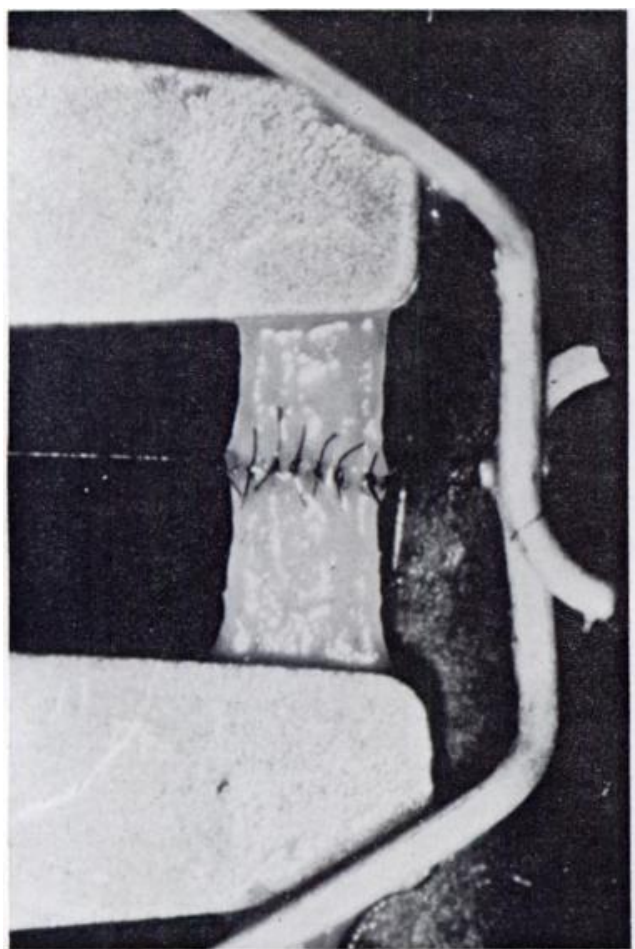

Fig. 5

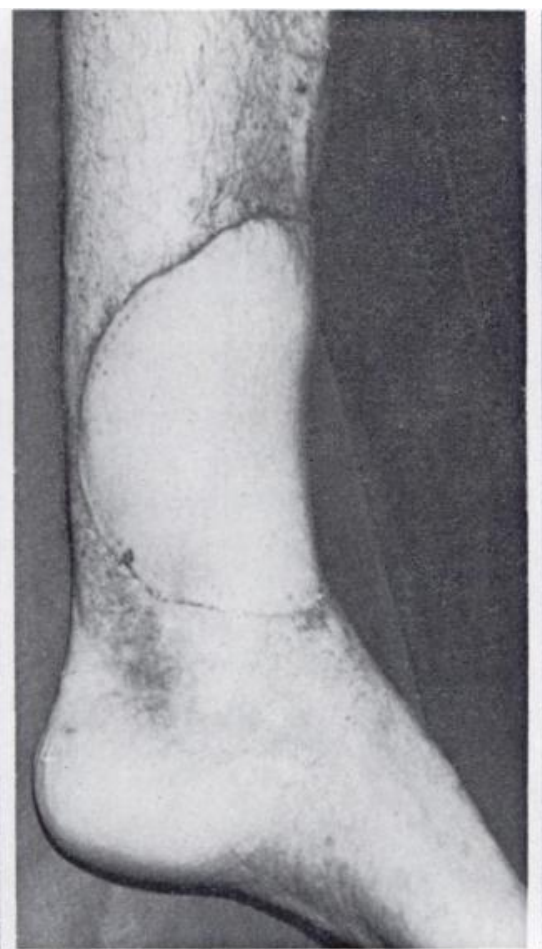

FIG. 6

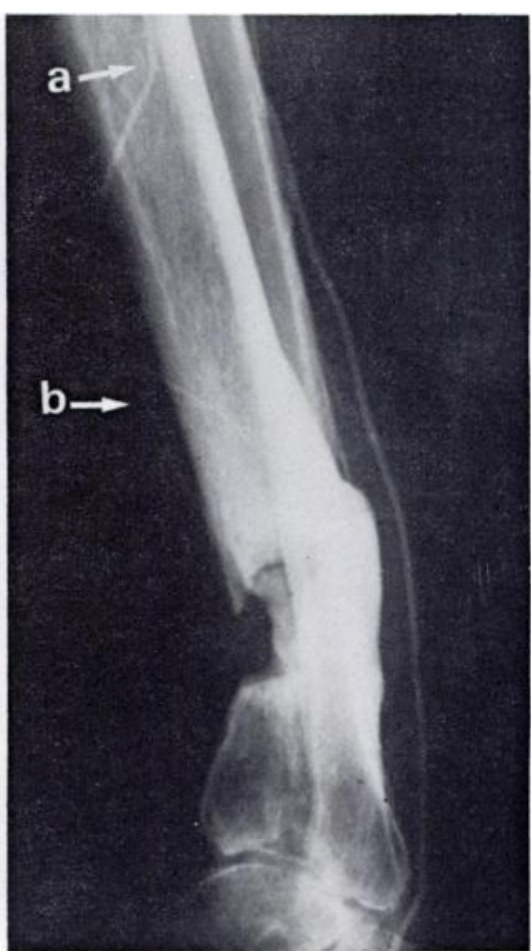

FIG. 7

Figure 5-Arterial anastomosis, using Canniesburn pattern approximator clamp and 11/0 monofilament nylon. Figure 6-The appearance of the flap four weeks after the operation. Figure 7-The arteriograph after operation, showing (a) sites of vein graft anastomoses and (b) course of superficial circumflex iliac artery in the transplanted flap.

Smith 1973). This has been attributed to the provision of a fresh blood supply. Compared with a free flap, a conventional flap has a relatively poor blood supply. A free flap has an abundant arterial supply, while a conventional flap is nourished only by capillary ingrowth. The provision of a greater blood supply to the fracture site is a further significant advantage of the free flap.

The principal disadvantage of free flap transfer is the length of the operation-in this case seven and a half hours. However, this is not substantially greater than the total operating time for a conventional multi-stage flap transfer. The operation is technically difficult. Special experience is required to anastomose small vessels reliably, and to deal with the intra-operative complications which can arise, such as spasm, thrombosis, leakage and failure of flow.

Microvascular surgery is likely to find a widening application in the orthopaedic field. Free flap transfer has been used successfully, not only for late repair, but also in the primary resurfacing of severe traumatic defects. This permits rapid mobilisation, and early secondary nerve and tendon repair.

For a high rate of success, procedures such as this are likely to remain the province of the specialist surgeon.

We wish to thank Mr T. B. Gardiner for his encouragement and for permission to describe this case, and Mr I. Binnie for his photographic assistance.

\section{REFERENCES}

Acland, R. (1972a) Prevention of thrombosis in microvascular surgery by the use of magnesium sulphate. British Journal of Plastic Surgery, 25, 292-299.

Acland, R. (1972b) A new needle for microvascular surgery. Surgery, 71, 130-131.

Connelly, J. R. (1948) Pedicle coverage in non-union of fractures. Plastic and Reconstructive Surgery, 3, 727-739.

Connelly, J. R. (1956) Plastic surgery in bone problems. Plastic and Reconstructive Surgery, 17, 129-167.

Daniel, R. K., and Taylor, G. I. (1973) Distant transfer of an island flap by microvascular anastomoses. Plastic and Reconstructive Surgery, 52, 111-117.

Grabb, W. C., and Smith, J. W. (1973) Plastic Surgery, pp. 924-925. Boston: Little, Brown and Company.

Harii, K., Ohmori, K., and Ohmori, S. (1974) Successful clinical transfer of ten free flaps by microvascular anastomoses. Plastic and Reconstructive Surgery, 53, 259-270.

Hayhurst, J. W., and O'Brien, B. McC. (1975) An experimental study of microvascular technique, patency rates and related factors. British Journal of Plastic Surgery, 28, 128-132.

McGregor, I. A., and Jackson, I. T. (1972) The groin flap. British Journal of Plastic Surgery, 25, 3-16.

Smith, P. J., Foley, B., McGregor, I. A., and Jackson, I. T. (1972) The anatomical basis of the groin flap. Plastic and Reconstructive Surgery, 49, $41-47$.

Stranc, M. F., Labandter, H., and Roy, A. (1975) A review of 196 tubed pedicles. British Journal of Plastic Surgery, 28, 54-58. 